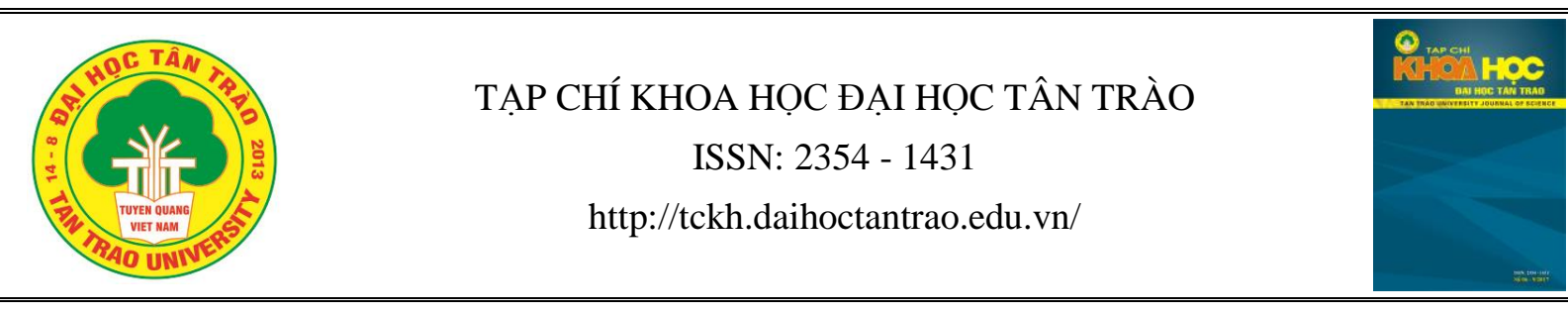

FACTORS AFFECTING THE DEVELOPMENT OF CAPACITY ORGANIZATION OF EXPERIENCE ACTIVITIES FOR STUDENTS OF PARTNERAL EDUCATION TAN TRAO UNIVERSITY

Ha My Hanh, ${ }^{1, *}$

${ }^{I}$ Tan Trao University, Vietnam

"Email address: hamyhanhedu@gmail.com

http://doi.org/10.51453/2354-1431/2021/626

\section{Article info}

Recieved: 03/8/2021

Accepted: 05/9/2021

\section{Keywords:}

Influencing factors, experimental activities, organizational capacity, students, Tan Trao University.

\begin{abstract}
This study is conducted to indicate the theoretical and practical basis of the influence of these factors on the development of the competency to organize creative experience activities for students in primary education at Tan University. The results of data analysis show that the most influencing factor is student activeness; Teaching competency, the competency of teachers to organize experience activities. Other factors such as facilities, learning materials; time; coordination among educational forces... has also had a lesser effect.
\end{abstract}


TẠP CHÍ KHOA HỌC ĐẠI HỌC TÂN TRÀO

ISSN: $2354-1431$

http://tckh.daihoctantrao.edu.vn/

\title{
CÁC YẾU TỐ ẢNH HƯởNG TỚI VIỆC PHÁT TRIỂN NĂNG LỰC TỔ CHỬC HOẠT ĐỘNG TRẢI NGHIỆM CHO SINH VIÊN NGÀNH GIÁO DỤC TIỂU HỌC TRƯờnG ĐẠI HỌC TÂN TRÀO
}

\author{
$H a \grave{a} M \tilde{y} H a n h^{1, *}$ \\ ${ }^{1}$ Truò̀ng Đại học Tân Trào, Việt Nam \\ *Địa chi email: hamyhanhedu@gmail.com \\ http://doi.org/10.51453/2354-1431/2021/626
}

\section{Thông tin bài viết}

Ngày nhận bài: 03/8/2021

Ngày duyệt đăng:05/9/2021

\section{Từ khóa:}

Yếu tố ảnh huởng, hoat động trải nghiệm, năng lục tổ chưc, sinh viên, Truờng Đại học Tân Trào.

\section{Tóm tắt}

Nghiên cứu này được thực hiện nhằm chỉ rõ cơ sở lý luận và thực tiễn mức độ ảnh hưởng của các yếu tố tới việc phát triển năng lực tổ chức hoạt động trải nghiệm cho sinh viên ngành giáo dục tiểu học trường Đại học Tân Trào. Kết quả phân thích số liệu cho thấy yếu tố gây ảnh hưởng cao nhất là tính tích cực hoạt động của sinh viên; năng lực dạy học, năng lực tổ chức hoạt động trài nghiệm của giáo viên. Các yếu tố khác như cơ sở vật chất, tài liệu học tập; thời gian; sự phối hợp giữa các lực lượng giáo dục...cũng có ảnh hưởng ở mức độ ít hơn.

\section{1. Đặt vấn đề}

Chương trình hoạt động trải nghiệm được chính thức ban hành kèm theo Thông tư số 32/2018/TTBGDĐT ngày 26 tháng 12 năm 2018 của Bộ trưởng Bộ Giáo dục và Đào tạo về việc ban hành chuoong trình giáo dục phổ thông'[3]. Theo đó, hoạt động trải nghiệm là hoạt động giáo dục bắt buộc trong trường tiểu học. Nó được thực hiện dựa trên sự huy động tổng hợp kiến thức và kỹ năng từ nhiều lĩnh vực giáo dục khác nhau nhằm hình thành những phẩm chất chủ yếu, năng lực chung và ba năng lực đặc thù của hoạt động này, đó là: năng lực thích ứng với cuộc sống, năng lực thiết kế và tổ chức hoạt động, năng lực định hướng nghề nghiệp.

Trường Đại học Tân Trào luôn quan tâm tới sự hình thành và phát triển năng lực người học đáp ứng chuẩn đầu ra. Trong những năm qua nhà trường đã có nhiều giải pháp hữu hiệu nhằm chỉnh sửa và phát triển chương trình đào tạo các ngành học đặc biệt là các mã ngành đại học chính quy. Thực hiện sự chỉ đạo của Ban giám hiệu nhà trường Bộ môn Giáo dục Tiểu học luôn nỗ lực, tích cực nghiên cứu chương trình giáo dục phổ thông mới, từ đó lựa chọn bổ sung học phần Tổ chức hoạt động trải nghiệm ở trường Tiểu học. Tuy nhiên, trong quá trình phát triển năng lực tổ chức hoạt động trải nghiệm cho sinh viên ngành giáo dục tiểu học chịu tác động bởi nhiều yếu tố. Việc xác định được mức độ ảnh hưởng của các yếu tố là cần thiết giúp cho việc phát triển năng lực tổ chức hoạt động trải nghiệm cho sinh viên đạt hiệu quả cao. 


\section{Nội dung}

\section{1. Đánh giá thục trạng các yếu tổ ảnh hưởng tới việc phát triển năng lục tổ chức hoạt động trải nghiệm cho sinh viên}

Năng lực tổ chức hoạt động trải nghiệm là sự tích hợp các kiến thức, kĩ năng, thái độ và kinh nghiệm cá nhân về lĩnh vực tổ chức hoạt động trải nghiệm, cho phép sinh viên thực hiện có kết quả việc tổ chức các hoạt động giáo dục theo hướng tăng cường trải nghiệm cho người học, trong các đợt thực tập và trong công việc ở trường phổ thông. Cụ thể:

2.1.1. Chuong trình đào tạo và chuẩn đầu ra của chuoong trình đào tạo

Chương trình đào tạo giáo viên tiểu học mô tả toàn bộ hoạt động trong một cơ sở giáo dục trong một thời gian xác định:

Khâu thiết kế chương trình.

Thực thi chương trình (dạy, học, kiểm tra đánh giá, nội khóa, ngoại khóa, thí nghiệm, dã ngoại,...).

Đánh giá cải tiến chương trình cho các khóa sau.

Mục tiêu của chương trình đào tạo giáo viên tiểu học là định hướng về kiến thức, kĩ năng, thái độ, năng lực mà người học, người dạy, nhà quản lí phải tuân thủ trong quá trình đào tạo.

Mục tiêu định hướng cho việc xác định nội dung đào tạo, phương thức đào tạo, và đánh giá kết quả đào tạo.

Chuẩn đầu ra $(\mathrm{CĐR})$ của chương trình đào tạo giáo viên là tổng hòa kiến thức, kĩ năng, thái độ, mà người học cần đạt được khi tốt nghiệp

CĐR của chương trình đào tạo trong kỉ nguyên thông tin phải được xây dựng trên cơ sở năng lực. Là "tổ hợp các hoạt động dựa trên sự huy động và sử dụng hiệu quả các nguồn kiến thức, kĩ năng khác nhau để giải quyết vấn đề (bao gồm cả kiến tạo kiến thức mới) hoặc có cách ứng xử phù hợp trong bối cảnh cuộc sống thực".

Đáp ứng yêu cầu của bậc học.

Đáp ứng yêu cầu của nghề nghiệp tương lai.

Đáp ứng yêu cầu phát triển của từng cá nhân.

CĐR là yếu tố quan trọng nhất quyết định việc:

Lựa chọn, tổ chức nội dung đào tạo giáo viên.

Định hướng tổ chức quá trình đào tạo giáo viên (phương thức đào tạo, hình thức tổ chức, phương pháp, phương tiện dạy học). Các hình thức tổ chức, phương pháp, phương tiện dạy học và kế hoạch kiểm tra đánh giá, kết quả đào tạo. Hình thức tổ chức trên lớp như: lớp đông, lớp nhỏ, seminar (các loại), làm việc nhóm, thực hành, thí nghiệm, diễn giải,... phải được sử dụng phù hợp với mục tiêu bài học (hướng tới $\mathrm{CĐR}$ ) với các phương pháp, phương tiện tương ứng. Hình thức tổ chức dạy tự học (ở nhà) phải được xem là hình thức tổ chức dạy học chính thức (được xếp vào thời khóa biểu), được dạy, học, kiểm tra đánh giá như các giờ khác.

Là chuẩn cho các hình thức kiểm tra đánh giá kết quả đào tạo giáo viên tiểu học.

CĐR liên quan đến chuyên môn, nghề nghiệp tương lai của $\mathrm{SV}$.

CĐR liên quan đến phẩm chất cá nhân và nghề nghiệp, năng lực tổ chức $\mathrm{HĐTN}$ của $\mathrm{SV}$ và các kĩ năng mềm.

Nếu mục tiêu của chương trình đào tạo giáo viên và $\mathrm{C} Đ R$ của chương trình đào tạo giáo viên được xác định rõ về năng lực tổ chức HĐTN cần đạt được ở người tốt nghiệp thì sẽ định hướng cho quá trình đào tạo, lựa chọn phương pháp, hình thức tổ chức dạy học và kiểm tra, đánh giá kết quả là những điều kiện có tính chất quyết định hình thành, phát triển năng lực tổ chức HĐTN ở SV ngành giáo dục tiểu học [4]

\subsubsection{Môi truờng giáo dục}

Sự phát triển năng lực tổ chức HĐTN cho SV ngành giáo dục tiểu học chỉ có thể được thực hiện trong một môi trường nhất định. Môi trường giáo dục tạo điều kiện để mỗi SV chiếm lĩnh được các kinh nghiệm xã hội loài người năng lực của bản thân nhằm phát triển nhân cách của mình.

Môi trường giáo dục ở Trường Đại học Tân Trào không chỉ ảnh hưởng tới quá trình dạy và học trong nhà trường mà còn ảnh hưởng tới quá trình phát triển năng lực tổ chức HĐTN cho SV ngành giáo dục tiểu học. Tuyên Quang là địa bàn sinh sống chủ yếu của các đồng bào dân tộc thiểu số Dao, Tày, Nùng, Mông, Cao Lan, Mường... Kinh tế gắn với sản xuất nông, lâm nghiệp, cuộc sống của các đồng bào dân tộc thiểu số gắn với tự nhiên với lao động thủ công, ít có điều kiện tiếp xúc với khoa học tiên tiến nên mức sống trung bình thấp; văn hóa đa dạng mỗi dân tộc có tiếng nói, chữ viêt và phong tục tập quán riêng. Phần lớn $\mathrm{SV}$ ngành giáo dục tiểu học thật thà, chất phát nhưng kĩ năng giao tiếp, ứng xử đặc biệt là tổ chức các hoạt động 
trải nghiệm vẫn còn lúng túng trước những tình huống mới nảy sinh điều này làm cho $\mathrm{SV}$ ngành giáo dục tiểu học hạn chế một số kĩ năng như thiết kế, xử lý tình huống trong tổ chức hoạt động và kĩ năng kiểm tra, đánh giá. Do vậy, cần phải xây dựng một môi trường giáo dục tích cực giúp các em có điều kiện tiếp xúc, giao lưu, trải nghiệm để phát triển năng lực tổ chức HĐTN đáp ứng yêu cầu thực tiễn.

Ngoài ra, môi trường giáo dục đại học trong trường ĐHTTr rất đa dạng và phong phú có ảnh hưởng tới sự phát triển năng lực tổ chức HĐTN cho $\mathrm{SV}$. Cụ thể: Cơ sở vật chất, cảnh quan của nhà trường cùng với các thiết bị dạy và học, điều kiện ăn ở là những yếu tố bên ngoài của người dạy và học nó có tác động mạnh mẽ tới các phẩm chất tâm lý, tới xúc cảm, niềm tin, hứng thú... tới việc phát triển tri thức, kĩ năng trong quá trình dạy học. Các hoạt động ngoài giờ lên lớp, hoạt động tập thể, hoạt động thực tiễn... trong môi trường giáo dục có tác động tích cực tới quá trình phát triển năng lực tổ chức HĐTN của $\mathrm{SV}$. Các mối quan hệ giao tiếp, ứng xử trong môi trường giáo dục nó tạo nên nét đặc trưng riêng của SV sư phạm (cách ăn, nói, mặc, ở, ứng xử). Đây vừa là nhân tố tạo nên môi trường giáo dục vừa là nhân tố có tác động mạnh mẽ tới nhân cách của $\mathrm{SV}$ đặc biệt là việc rèn luyện các kĩ năng giao tiếp, kĩ năng ứng xử... Do vậy, môi trường giáo dục thuận lợi sẽ là điều kiện tốt giúp SV năng lực tổ chức HĐTN và ngược lại.

Tuy nhiên, tính chất và mức độ ảnh hưởng của môi trường đối với sự phát triển nhân cách nói chung và sự phát triển năng lực tổ chức HĐTN nói riêng còn tùy thuộc vào lập trường, quan điểm, thái độ của từng $\mathrm{SV}$ đối với các ảnh hưởng đó, cũng như tùy thuộc vào xu hướng và năng lực, vào mức độ từng $\mathrm{SV}$ tham gia cải biến môi trường đó. [4]

2.1.3. Năng lực dạy học, năng lực tổ chưc HĐTN của $G V$

Năng lực là nhân tố quan trọng quyết định tới thành công của mỗi hoạt động. Năng lực dạy học được hiểu là "thuộc tính của người $\mathrm{GV}$ tổ chức hoạt động dạy học một cách có hiệu quả (đảm bảo các mục tiêu dạy học về kiến thức, kĩ năng, thái độ và sự phát triển năng lực bằng sự chuyên nghiệp và tính trách nhiệm) bao gồm năng lực thiết kế dạy học, tiến hành dạy học, đánh giá dạy học và quản lí dạy học". Năng lực dạy học của GV trong đó bao hàm kiến thức, kĩ năng chuyên môn, năng lực tổ chức các hoạt động cho SV trải nghiệm thực tế để chiếm lĩnh tri thức, hình thành kĩ năng là yếu tố ảnh hưởng trực tiếp tới quá trình phát triển năng lực tổ chức HĐTN cho SV.

Để phát triển năng lực tổ chức HĐTN cho SV người GV phải có năng lực quản lý để xây dựng kế hoạch hoạt động, tổ chức, chỉ đạo và kiểm tra, đánh giá các hoạt động của $\mathrm{SV}$ nhằm bồi dưỡng cho $\mathrm{SV}$ hệ thống kiến thức, kĩ năng tổ chức HĐTN và các phẩm chất tâm lý cần thiết giúp SV ngày một hoàn thiện nhân cách của mình đáp ứng với yêu cầu của xã hội. Chính vì vậy, nếu $\mathrm{GV}$ có năng lực tổ chức HĐTN tốt là nhân tố giữ vai trò chủ đạo định hướng, chỉ đạo cho quá trình phát triển năng lực tổ chức HĐTN của $\mathrm{SV}$. Ngược lại, nếu GV không có năng lực năng lực tổ chức HĐTN sẽ hạn chế sự phát triển năng lực tổ chức HĐTN của $\mathrm{SV}$.

\subsubsection{Tính tích cực hoạt động của SV}

Nhân cách có thể coi là hoàn thiện khi nó đáp ứng yêu cầu xã hội hiện đại. Xã hội luôn phát triển, con người phải luôn tự điều chỉnh nhân cách của mình theo yêu cầu mới của xã hội, đó là sự hoàn thiện nhân cách. Sự hoàn thiện nhân cách diễn ra thông qua việc cá nhân tự ý thức, tự rèn luyện, tự giáo dục, tự học, tự bồi dưỡng không ngừng. .

Tích cực hoạt động của SV ngành giáo dục tiểu học giúp SV tìm tòi khám phá ra những vấn đề mới, giúp $\mathrm{SV}$ hiểu rõ bản chất của vấn đề một cách sâu sắc nhất, đặc biệt tính tích cực của $\mathrm{SV}$ giúp $\mathrm{SV}$ củng cố tri thức, rèn luyện các phẩm chất và các kĩ năng tổ chức HĐTN một cách tốt nhất và hiệu quả nhất. Không ít những SV tuy có đầy đủ mọi điều kiện để học tập như: môi trường giáo dục thuận lợi, thầy giỏi, tài liệu hay, cơ sở vật chất đầy đủ... nhưng năng tổ chức HĐTN của họ vẫn kém. Do vậy, có thể nói tính tích cực hoạt động của $\mathrm{SV}$ là yếu tố quyết định trực tiếp sự hình thành và phát triển nhân cách nói chung và năng tổ chức HĐTN nói riêng. Chính vì thế, đối với người $\mathrm{GV}$ phải biến quá trình dạy học, giáo dục thành quá trình tự học, tự giáo dục ở $\mathrm{SV}$ và coi trọng việc xây dựng nhu cầu, động cơ, hứng thú, mục đích hoạt động cho SV.

2.2. Thục trạng các yếu tố ảnh hương tới việc phát triển năng lục tổ chức hoạt động trải nghiệm 
cho sinh viên ngành giáo dục tiểu học trưòng Đại học Tân Trào

\subsubsection{Mục tiêu khảo sát}

- Thu thập tình hình khách quan, số liệu thực tế về yếu tố ảnh hưởng tới việc phát triển năng lực tổ chức hoạt động trải nghiệm cho sinh viên ngành giáo dục tiểu học Trường Đại học Tân Trào

- Khái quát hóa, phân tích và đánh giá tình hình thực trạng các yếu tố ảnh hưởng tới việc phát triển năng lực tổ chức hoạt động trải nghiệm cho sinh viên ngành giáo dục tiểu học Trường Đại học Tân Trào

- Từ đó rút ra những thuận lợi, khó khăn, rào cản và nguyên nhân của các yếu tố ảnh hưởng tới việc phát triển năng lực tổ chức hoạt động trải nghiệm cho sinh viên ngành giáo dục tiểu học Trường Đại học Tân Trào

\subsection{2. Đối tượng khảo sát}

Tiến hành khảo sát các đối tượng $\mathrm{GV}, \mathrm{SV}$ ngành GDTH thuộc trường Đại học Tân Trào cụ thể:

- Tổng số GV được điều tra là 30 người. Phần lớn số $\mathrm{GV}$ được điều tra là những người có kinh nghiệm giảng dạy, có trình độ từ thạc sĩ trở lên.

- Tổng số SV được điều tra là 156 người. Sau khi sàng lọc loại bỏ những phiếu không đảm bảo yêu cầu điều tra còn lại đã đưa vào xử lý 152 phiếu.

\subsubsection{Phương pháp khảo sát}

- Khảo sát bằng phiếu hỏi: để điều tra $\mathrm{GV}$ và $\mathrm{SV}$ trường Đại học Tân Trào theo những nội dung được thể hiện qua phiếu khảo sát

- Phuoong pháp quan sát su phạm: quan sát các hoạt động giáo dục, dạy học, hoạt động thực tế, hoạt động Đoàn, Đội và Hội SV...

- Phuơng pháp phỏng vấn: Tiến hành phỏng vấn $\mathrm{GV}, \mathrm{SV}$ để làm rõ những vấn đề mà bảng hỏi và quan sát chưa thu thập được.

\subsubsection{Kết quả khảo sát}

\subsubsection{Kết quả khảo sát ở giáo viên}

Từ bảng 1 cho thấy phần lớn $\mathrm{GV}$ đều đánh giá những yếu tố trên có ảnh hưởng rất cao tới việc phát triển năng lực tổ chức HĐTN của $\mathrm{SV}$, trong đó yếu tố gây ảnh hưởng lớn nhất là tính tích cực hoạt động của $\mathrm{SV}$ (chiếm 83,3\%) xếp thứ nhất; Năng lực dạy học, năng lực tổ chức HĐTN của GV (chiếm $80 \%$ ) xếp thứ hai; Chương trình đào tạo và $\mathrm{CĐR}$ của chương trình đào tạo (chiếm 76,7\%) xếp thứ ba; Môi trường giáo dục (chiếm 69\%) xếp thứ tư. Ở mức độ tương đối cao Các yếu tố khác như cơ sở vật chất, tài liệu học tập; thời gian; sự phối hợp giữa các lực lượng giáo dục... (chiếm 40\%). Ở mức độ trung bình các yếu tố khác chiếm $60 \%$; môi trường giáo dục (chiếm 3,4\%). Như vậy, các yếu tố trên đều có ảnh hưởng tới việc phát triển năng lực tổ chức HĐTN của $\mathrm{SV}$, tuy nhiên mức độ ảnh hưởng của các yếu tố là khác nhau.

Bảng 1: Ý kiến của GV về những yếu tố ảnh hưởng đến việc phát triển năng lực tổ chức HĐTN

\begin{tabular}{|c|c|c|c|c|c|c|c|c|c|c|}
\hline \multirow{3}{*}{ Những yếu tố ảnh hưởng } & \multicolumn{10}{|c|}{ Mức độ } \\
\hline & \multicolumn{2}{|c|}{ Cao } & \multicolumn{2}{|c|}{$\begin{array}{l}\text { Tương đối } \\
\text { cao }\end{array}$} & \multicolumn{2}{|c|}{ Trung bình } & \multicolumn{2}{|c|}{$\begin{array}{l}\text { Tương đối } \\
\text { thấp }\end{array}$} & \multicolumn{2}{|c|}{ Thấp } \\
\hline & SL & $\%$ & SL & $\%$ & SL & $\%$ & SL & $\%$ & SL & $\%$ \\
\hline $\begin{array}{l}\text { 1. Chương trình đào tạo và } \\
\text { CĐR của chương trình đào } \\
\text { tạo }\end{array}$ & 23 & 76,7 & 7 & 23,3 & 0 & $\mathbf{0 , 0}$ & 0 & $\mathbf{0}$ & 0 & $\mathbf{0}$ \\
\hline 2. Môi trường giáo dục & 20 & 69,0 & 8 & 27,6 & 1 & 3,4 & 0 & $\mathbf{0}$ & 0 & $\mathbf{0}$ \\
\hline $\begin{array}{l}\text { 3. Năng lực dạy học, năng } \\
\text { lực tổ chức HĐTN của GV }\end{array}$ & 24 & 80,0 & 6 & 20,0 & 0 & $\mathbf{0 , 0}$ & 0 & $\mathbf{0}$ & 0 & $\mathbf{0}$ \\
\hline $\begin{array}{l}\text { 4. Tính tích cực hoạt động của } \\
\text { SV }\end{array}$ & 25 & 83,3 & 5 & 16,7 & 0 & $\mathbf{0 , 0}$ & 0 & $\mathbf{0}$ & 0 & $\mathbf{0}$ \\
\hline 5. Yếu tố khác... & 0 & $\mathbf{0 , 0}$ & 12 & 40,0 & 18 & 60,0 & 0 & $\mathbf{0}$ & 0 & $\mathbf{0}$ \\
\hline
\end{tabular}

Bên cạnh khảo sát bằng bảng hỏi tôi còn tiến hành phỏng vấn sâu 1 số GV thông qua câu hỏi: Tại 
sao thầy/cô lại cho rằng tính tích cực hoạt động của SV có ảnh hương lón đến việc phát triển năng lực tổ chúc HĐTN cho SV?

Kết quả phỏng vấn cho thấy $5 / 5 \mathrm{GV}$ được hỏi đều cho rằng tính tích cực hoạt động của $\mathrm{SV}$ có ảnh hưởng lớn đến việc phát triển năng lực tổ chức HĐTN bởi vì nếu sinh viên không tích cực, tự giác, không muốn tiếp nhận các kiến thức, kĩ năng, thái độ thì cho dù có tổ chức các hoạt động phong phú, đa dạng hay được xác định trong chuẩn đầu ra của chương trình đào tạo... thì những yếu tố này không giữ vai trò quyết định đến việc phát triển năng lực tổ chức $\mathrm{HĐTN}$ cho $\mathrm{SV}$ mà yếu tố quyết định chính là tính tích cực hoạt động của $\mathrm{SV}$.

\subsubsection{Kết quả khảo sát ở sinh viên}

Từ bảng 2 cho thấy ý kiến đánh giá của $\mathrm{SV}$ và GV mặc dù có sự khác biệt nhưng họ đều đánh giá những yếu tố trên có ảnh hưởng rất cao tới việc phát triển năng lực tổ chức HĐTN của $\mathrm{SV}$, trong đó yếu tố gây ảnh hưởng lớn nhất là tính tích cực hoạt động của SV (chiếm 84,2\%) xếp thứ nhất; Năng lực dạy học, năng lực tổ chức HĐTN của GV (chiếm $79,6 \%$ ) xếp thứ hai; Chương trình đào tạo và $\mathrm{CĐR}$ của chương trình đào tạo (chiếm 76,3\%) xếp thứ ba; Môi trường giáo dục (chiếm $65,1 \%$ ) xếp thứ tư. Ở mức độ tương đối cao Các yếu tố khác (chiếm $37,3 \%$ ). Ở mức độ trung bình các yếu tố khác (chiếm 62,7\%); môi trường giáo dục (chiếm 5,9\%).

Bảng 2: Ý kiến của $S V$ về những yếu tố ảnh hưởng đến việc phát triển năng lực tổ chức HĐTN

\begin{tabular}{|c|c|c|c|c|c|c|c|c|c|c|}
\hline \multirow{3}{*}{ Những yếu tố ảnh hưởng } & \multicolumn{10}{|c|}{ Mức độ } \\
\hline & \multicolumn{2}{|c|}{ Cao } & \multicolumn{2}{|c|}{$\begin{array}{l}\text { Tương đối } \\
\text { cao }\end{array}$} & \multicolumn{2}{|c|}{ Trung bình } & \multicolumn{2}{|c|}{$\begin{array}{l}\text { Tương đối } \\
\text { thấp }\end{array}$} & \multicolumn{2}{|c|}{ Thấp } \\
\hline & SL & $\%$ & SL & $\%$ & SL & $\%$ & SL & $\%$ & SL & $\%$ \\
\hline $\begin{array}{l}\text { 1. Chương trình đào tạo và } \\
\text { CĐR của chương trình đào } \\
\text { tạo }\end{array}$ & 116 & 76,3 & 36 & 23,7 & 0 & $\mathbf{0}$ & 0 & $\mathbf{0}$ & 0 & $\mathbf{0}$ \\
\hline 2. Môi trường giáo dục & 99 & 65,1 & 44 & 28,9 & 9 & $\mathbf{5 , 9}$ & 0 & $\mathbf{0}$ & 0 & $\mathbf{0}$ \\
\hline $\begin{array}{l}\text { 3. Năng lực dạy học, năng } \\
\text { lực tổ chức HĐTN của GV }\end{array}$ & 121 & 79,6 & 31 & 20,4 & 0 & $\mathbf{0 , 0}$ & 0 & $\mathbf{0}$ & 0 & $\mathbf{0}$ \\
\hline $\begin{array}{l}\text { 4. Tính tích cực hoạt động của } \\
\text { SV }\end{array}$ & 128 & 84,2 & 24 & 15,8 & 0 & $\mathbf{0 , 0}$ & 0 & $\mathbf{0}$ & 0 & $\mathbf{0}$ \\
\hline 5. Yếu tố khác... & 0 & $\mathbf{0 , 0}$ & 56 & 37,3 & 94 & 62,7 & 0 & $\mathbf{0}$ & 0 & $\mathbf{0}$ \\
\hline
\end{tabular}

Qua quan sát hoạt động dạy học và giáo dục trong nhà trường tôi nhận thấy những $\mathrm{SV}$ tích cực thì phát triển rất tốt về kiến thức, kĩ năng, thái độ tổ chức các HĐTN. Ngược lại, có những $\mathrm{SV}$ được tạo điều kiện rất tốt để được tham gia vào thiết kế, tổ chức các hoạt động nhưng lại chưa sáng tạo, chủ động đều này có ảnh hưởng trực tiếp đến kế quả của hoạt động.

Đánh giá chung: Tất cả những yếu tố trên đều có ảnh hưởng rất lớn tới việc phát triển năng lực tổ chức HĐTN của $\mathrm{SV}$, trong đó yếu tố gây ảnh hưởng cao nhất là tính tích cực hoạt động của $\mathrm{SV}$; Năng lực dạy học, năng lực tổ chức HĐTN của GV. Các yếu tố khác như cơ sở vật chất, tài liệu học tập; thời gian; sự phối hợp giữa các lực lượng giáo dục...cũng có ảnh hưởng nhưng ở mức độ thâp hơn. Do vậy, trong dạy học và giáo dục người $G V$ cần chú ý quan tâm đổi mới phương pháp nhằm phát huy tối đa năng lực, sở trường của từng cá nhân đồng thời cần chú ý tới hứng thú của tập thể sinh viên.

\section{Kết luận}

Hoạt động trải nghiệm chính thức được đưa vào giảng dạy chương trình giáo dục phổ thông mới do vậy việc tìm hiểu các yếu tổ ảnh hưởng tới việc phát triển năng lực tổ chức hoạt động trải nghiệm cho sinh viên là việc làm rất cần thiết vì nhằm nâng cao hiệu quả phát triển năng lực tổ chức HĐTN cho sinh viên ngành giáo dục tiểu học. 
Kết quả khảo sát từ phiếu hỏi, phỏng vấn và quan sát cho thấy việc phát triển năng lực tổ chức HĐTN cho SV ngành giáo dục Tiểu học chịu tác động bởi nhiều yếu tố, trong đó yếu tố gây ảnh hưởng nhiều nhất là tính tích cực hoạt động của SV; Năng lực dạy học, năng lực tổ chức HĐTN của GV. Các yếu tố khác như cơ sở vật chất, tài liệu học tập; thời gian; sự phối hợp giữa các lực lượng giáo dục...cũng có ảnh hưởng nhưng ở mức độ trung bình.

\section{REFERENCES}

[1] Ministry of Education and Training. (2018). General education program, Experiential activities and experiential activities, career guidance.

[2] Development strategy project of Tan Trao University in the period of 2018 - 2025, orientation to 2030 .

[3] Circular No. 32/2018/TT-BGDDT dated December 26, 2018 of the Minister of Education and Training on the Promulgation of the general education program.

[4] Hanh, H. M. (2016). Developing capacity for social activities for students of pedagogical universities in the northern mountainous region in training under the credit system. Thai Nguyen University Publishing House.

[5] Tan Trao University. (2018). Decision No. 03/NQ-HDT, dated December 21, 2018 Tan Trao University development strategy for the period 2018-2025, with orientation to 2030 .

[6] Lien, N. T. (2016). Organize creative experiential activities in high schools. Education Publishing House, Hanoi.

[7] Kien, H. C., Hang, N. T. T., Hong, D. T. B., Thanh, D. (2020). Design experience activities with the theme "Creating Art" for middle school students in the career-oriented experience program. Education Journal, 490:49-53. 